\title{
Rectification and Spin Filtering Effects in Benzene Molecules Connected Via Co and N Atoms
}

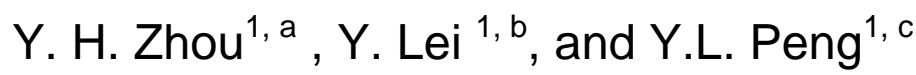 \\ ${ }^{1}$ Department of Information Engineering, Gannan Medical University, Ganzhou, Jiangxi, China \\ ayhzhou80@gmu.cn, 'reiyin80@163.com, cylpeng6124@gmail.com
}

Keywords: phenyl-ring, multi-functional device, spins transport properties, nonequilibrium Green's functions.

\begin{abstract}
The transport properties in molecule consisting of two phenyl-rings connected via Co and $\mathrm{N}$ atoms are investigated by first principles, where the molecule is put between two ZGNR electrodes. Rectification and spin filtering effects are found in our designed molecule. The current at positive biases is much bigger than that at negative biases for both the P and AP configurations, which results in rectification effect. In addition, the spin down current (the spin up current) is nearly depressed for the (anti) configuration, while the spin up current (the spin down current) owns comparable values. So spin filter can be achived. Detailed explanation via transmission spectra is given to the above useful phenomenon.
\end{abstract}

\section{Introduction}

It is of great interest in studying the electron transport through individual molecules for the purpose of using molecules as components in molecular-scale circuit, which can greatly decrease the size of present electron devices, while simulously increase the working velosity of electron device. In recent years, different types of molecular devices have been studied experimentally and theoretically [1][2]. And lots of works related to organic molecular devices have been done and many interesting phenomena based on organic molecular devices have been attained. For example, molecular rectifiers, switchers and transistors, which is the properties of classical electron device, have been designed and investigated widely [3][4]. Previous studies showed that the conductance of the phenyl rings are influenced significantly by the conformation, and therefore, the phenyl rings can show the switching effect by changing the conformation such as rotating the ring from coplanar case to perpendicular case [5]. For example, in the year 2007, the spin-polarized transport properties of the 1, 12-tri-benzene-dithiolate molecule coupled to two magnetic nickel contacts have been studied, and the magneto-transport properties were found to be very sensitive to deformations of one of the three benzene rings is rotated from the equilibrium position [6]. In our present work, we found magnetoresistance and fine spin filtering effects in a device of Co-phthalocyanine connected to two zigzag graphene nanoribbon electrondes(ZGNR) [7]. Since the graphene sheet was fabricated experimentally in 2004, lots of studies reveal that the ZGNR is a good candidate for electrodes in spintronic devices [8]. The zigzag-edged GNRs can be non-magnetic, antiferromagnetic or ferromagnetic states depending upon the external means. Under an external magnetic fields, two electrodes can point in the same or opposite direction, the ZGNR electrodes can show the $(P)[1,1]$ or antiparalllel (AP) [1,-1] spin configuration. Besides, dual spin filtering effect was shown in 8-ZGNR (the number 8 indicates the width of the ZGNR) but not in a 7-ZGNR [9], which can be used for devising some molecular devices like molecular bipolar spin diodes. Further, the spintronic molecular device based on phenyl rings connected by Co and $\mathrm{N}$ atoms has not been reported yet. So in present work, we study the transport properties in the molecule of two phenyl-rings connected via Co and $\mathrm{N}$ atoms by first principles. We find rectification and spin filtering effects our designed molecule. For example, the current at positive biases is much bigger than that at negative biases for both the $\mathrm{P}$ and AP configurations, which results in rectification effect. And the spin down current (the spin up current) is nearly depressed for the (anti) configuration, while the spin up current (the spin down current) owns comparable values, which demonstrates good spin filtering effect. 


\section{Simulation Model and Calculation Method}

Fig. 1 shows the molecular device model that the two phenyl rings connected by Co and $\mathrm{N}$ atoms are sandwiched between 8-ZGNR electrodes. The molecular device is divided into three parts: left electrode, the scattering central region and the right electrode. The central region contains two repeated ZGNR unit cells in the left electrodes and two repeated ZGNR unit cells in the right electrodes indicated by $\mathrm{C}$, and both the left and right electrodes are described by a supercell with two repeated ZGNR unit cells (color in blue and in purple). We model a supercell with a large enough vacuum layer in $\mathrm{x}$ and $\mathrm{y}$ directions in order to eliminate the interaction between ZGNRs in neighboring cells. The calculations are done by a developed first-principles software package Atomistix Toolkit (ATK) based on the density functional theory combined with nonequilibrium Greens functions (DFT/NEGF) [10]. In the calculation, the local spin density approximation (LSDA) is used for exchange-correlation potential. The core electrons are described by norm-conserving pseudopotentials. The cutoff energy of 150 Ry and a Monkhorst-Pack K-mesh of $(1,1,150)$ are used. The convergence criterion for Hamiltonian and the electron density are $10^{-5}$. The single-zeta polarized (SZP) basis set is adopted for electron wave function. All atomic positions are fully relaxed using quasi-Newton method until the force tolerance to $0.02 \mathrm{eV} / \AA$.



Fig. 1. (Color online) The device model: two phenyl rings connected by Co and $\mathrm{N}$ atoms are sandwiched between 8-ZGNR electrodes. The letters L, C and R represent the left electrode, the scattering central region and right electrode, respectively.

\section{Results and Discussions}

Fig. 2 shows the I-V curves for P and AP conformations of the designed curcuit. The current at certain positive biases is much bigger than that at negative biases for both the $\mathrm{P}$ and AP configurations, which results in rectification effect. Furthur, with the increase of the positive bias, the current for both the P and AP conformations firstly increases and then decrease, which leads to negative differential resistance (NDR). In addition,as shown in Fig. 3, spin filters are achived. The spin down current (the spin up current) is nearly depressed for the P (AP) configuration, while the spin up current (the spin down current) owns comparable values. In the following, we give explanation via transmission spectra to the above useful phenomenon.

The urrent through the system is obtained by integrating the transmission function in the bias window using the Landauer-Büttiker formula. So the value of current depends on the transmision in the bias window. So we show the transmission spectra correspont to the peak of the I-V curves that for the P configuration at the bias $0.1 \mathrm{~V}$ (a) and for the AP configuration at the bias $0.4 \mathrm{~V}$ (b). The spin 


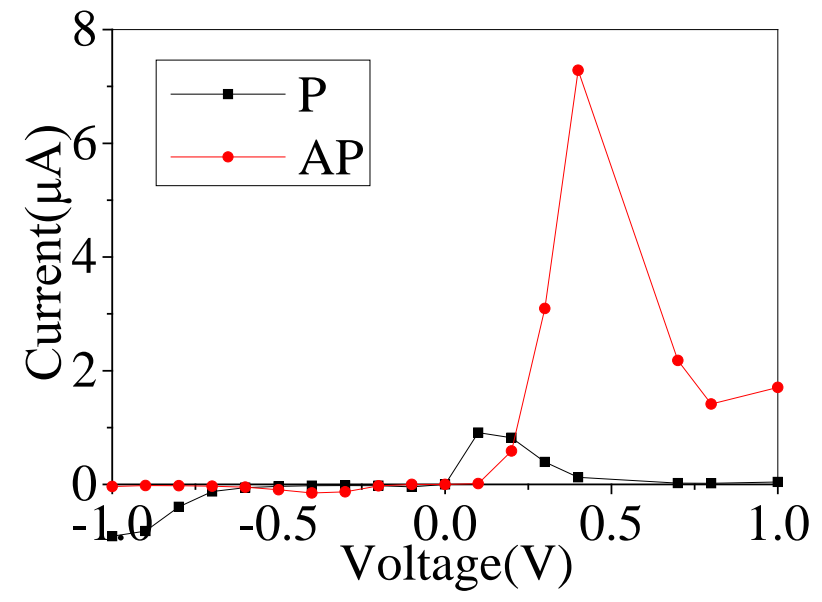

Fig. 2. (Color online) The I-V curves for $\mathrm{P}$ and AP conformations of the designed curcuit. The black line is for the $\mathrm{P}$ configuration and the red line for the AP configuration.



Fig. 3. (Color online) The spin-resolved I-V curves for the disigned curcuit. (a) for the $P$ configuration and (b) for the AP configuration. The black line indicate the spin up current and the red line indicate the spin down current.

down transmission for the P configuration and the spin up transmission for the AP configuration in the bias window are degenarated. So the total current for $\mathrm{P}$ depends on the spin up transmission and that for AP configuration depends on the spin down transmission. Thus fine spin filtering effect happens. The spin up and spin down transmission coefficient in the bias window at negative bias is nearly zero for both the P and AP configurations, which is not shown here, but can infered from Fig. 4. That is: with the increase of the bias, the transmission at equilibrium will move toward left, and with the decrease of the bias, the transmission at equilibrium will move toward right. So at negative bias, the current for both the $\mathrm{P}$ and AP configurations are nearly zero. And then rectification happens in both the $\mathrm{P}$ and AP configurations.

\section{Summary}

In the manuscrit, we studied the transport properties in molecular devices consisting of two phenyl-rings connected via Co and $\mathrm{N}$ atoms by first principles. Rectification and spin filtering effects are found in our designed molecule. Rectification and spin filtering effects were gained in our designed molecule. The current at positive biases is much bigger than that at negative biases for both the $\mathrm{P}$ and AP configurations, which leads to rectification effect. In addition, the spin down current (the spin up current) is nearly depressed for the (anti) configuration, while the spin up current (the spin down current) owns comparable values, which leads to spin filter can be achived. We gave an explanation to the above useful phenomenon via transmission spectra. 



Fig. 4. (Color online) The energy dependent spin-up (the blue line) and spin-down (the red line) transmission spectra. (a) for the P configuration at the bias $0.1 \mathrm{~V}$ and (b) for the AP configuration at the bias $0.4 \mathrm{~V}$.

\section{Acknowledgment}

This work was supported by the National Natural Science Foundation of China, (No. 61361001), by Natural Science Foundation of Jiangxi Province(No. 20132BAB211033), by Natural Science Foundation of Jiangxi Province(No. 20122BAB212007), China and by the Doctoral graduate innovation fund of Hunan Province (No. 521293040), China.

\section{References}

[1] E. Malic, C. Weber, M. Richter, V. Atalla, T. Klamroth, P. Saalfrank, S. Reich, A. Knorr, Microscopic Model of the Optical Absorption of Carbon Nanotubes Functionalized with Molecular Spiropyran Photoswitches [J]. Phys. Rev. Lett. 2011 (106) 097401

[2] M. G. Vergniory, J. M. Granadino-Roldan, A. Garcia-Lekue, L. W. Wang, Molecular conductivity switching of two benzene rings under electric field [J]. Appl. Phys. Lett. 2010 (97) 262114-3.

[3] T. Ozaki, K. Nishio, H. Weng, H. Kino, Dual spinfiltereffect in a zigzaggraphenenanoribbon [J]. Phys. Rev. B 2010 (81) 075422.

[4] M. C. McCarthy, M. J. Travers, A. Kovacs, W. Chen, S. E. Novick, C. A. Gottlieb, P. Thaddeus, Detection and Characterization of the Cumulene Carbenes H2C5 and H2C6 [J]. Science 1997 (275) 518-520.

[5] H. Q. Wan, B. H. Zhou, X. W. Chen, C. Q. Sun, G. H. Zhou, Switching, Dual Spin-Filtering Effects, and Negative Differential Resistance in a Carbon-Based Molecular Device [J]. J. Phys. Chem. C 2012 (116) 2570-2574.

[6] L. Senapati, R. Pati, S. C. Erwin, Controlling spin-polarized electron transport through a molecule: The role of molecular conformation [J]. Phys. Rev. B 2007 (76) 024438.

[7] Y. H. Zhou, J. Zeng, L. M. Tang, K. Q. Chen, W. P. Hu, Giant magnetoresistance effect and spin filters in phthalocyanine-based molecular devices [J]. Org. Electron. 2013 (14) 2940-2947.

[8] K. S. Novoselov, A. K. Geim, S. V. Morozov, D. Jiang, Y. Zhang, S. V. Dubonos, I. V. Grigorieva, A. A. Firsov, Electric field effect in atomically thin carbon films [J]. Science 2004 (306) 666-669.

[9] M. G. Zeng, L. Shen, M. Zhou, C. Zhang, Y. P. Feng, Graphene-based bipolar spin diode and spin transistor: Rectification and amplification of spin-polarized current [J]. Phys. Rev. B 2011 (83) 115427. 
[10] M. Brandbyge, J. L. Mozos, P. Qrdejon, J. Taylor, K. Stokbro, Density-functional method for nonequilibrium electron transport [J]. Phys. Rev. B 2002 (65) 165401. 